\title{
Synthesis, Characterization and Antibacterial Activity of Novel Pyridones
}

\author{
Hayreddin Gezegen ${ }^{\mathrm{a}}$, Mustafa Ceylan ${ }^{\mathrm{a},{ }^{*}}$, İsa Karaman ${ }^{\mathrm{b}}$, Ertan Şahin $^{\mathrm{c}}$ \\ ${ }^{a}$ Department of Chemistry, Faculty of Arts and Sciences, Gaziosmanpasa University, 60250 Tokat, Turkey \\ ${ }^{\mathrm{b}}$ Department of Biology, Faculty of Arts and Sciences, Gaziosmanpasa University, 60250 Tokat, Turkey \\ ${ }^{c}$ Department of Chemistry, Faculty of Science, Atatürk University, 25240 Erzurum, Turkey \\ E-mail address: mustafac.ceylan@gop.edu.tr
}

\section{Supplemental Materials}

Table S1. Minimum-inhibitory concentrations (MIC, in $\mu \mathrm{g} / \mathrm{ml}$ ) of synthesized

\begin{tabular}{|c|c|c|c|c|}
\hline \multicolumn{5}{|c|}{ Microorganisms } \\
\hline Compounds & $\begin{array}{c}\text { Staphylococcus } \\
\text { aureus } \\
\text { ATCC } 29213\end{array}$ & $\begin{array}{c}\beta \text {-Hemolytic } \\
\text { Streptococcus } \\
\text { ATCC } 2957\end{array}$ & $\begin{array}{c}\text { Escherichia } \\
\text { coli } 111\end{array}$ & $\begin{array}{c}\text { Salmonella } \\
\text { Enteritidis } \\
\text { ATCC } 13076\end{array}$ \\
\hline $4 a$ & 250 & 250 & 250 & 250 \\
\hline $4 b$ & 250 & 250 & 250 & 250 \\
\hline 4c & 250 & 250 & 250 & 250 \\
\hline 4d & 250 & 250 & 250 & 250 \\
\hline $4 e$ & 125 & 250 & 250 & 125 \\
\hline $5 a$ & 250 & 250 & 125 & 250 \\
\hline $5 \mathbf{b}$ & 250 & 250 & 125 & 125 \\
\hline $5 c$ & 250 & 250 & 125 & 125 \\
\hline $5 d$ & 250 & 250 & 125 & 125 \\
\hline PEN & 31.25 & $>250$ & 1.95 & 0.98 \\
\hline CEF & 31.25 & 15.63 & 31.25 & 62.25 \\
\hline
\end{tabular}

PEN: Penicillin G CEF: Ceftriaxone

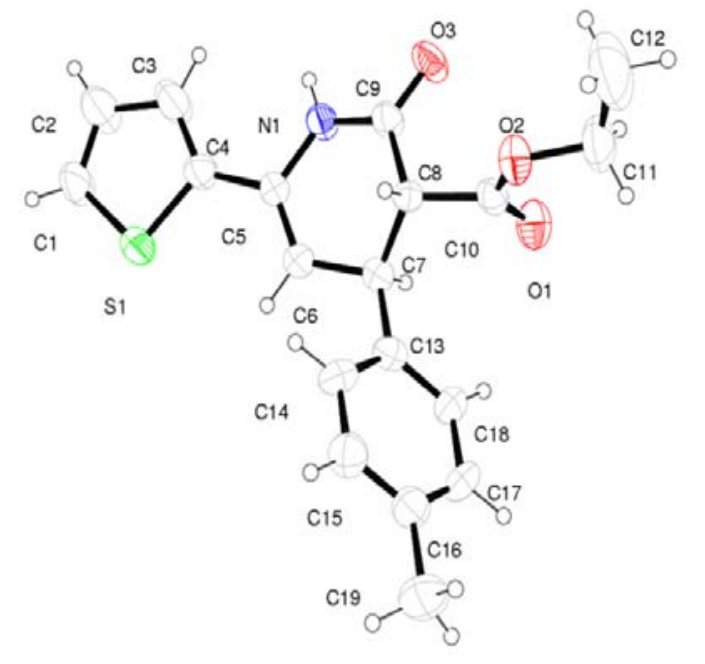

Figure S1. ORTEP drawing of the molecule $\mathbf{4 b}$. Thermal ellipsoids are shown at 50\% probability level. 


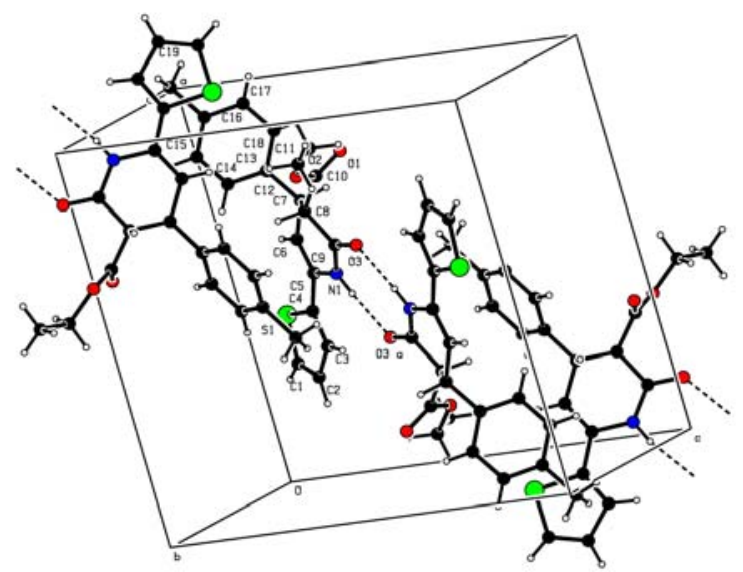

Figure S2. $H$-bonded dimers lie (dashed lines) along diagonal axis in the unit cell. $\mathrm{N} 1-\mathrm{H} \cdots \mathrm{O} 3^{\mathrm{a}}=2.900(6) \AA$, $<\left(\mathrm{N} 3-\mathrm{H} 3 \cdots \mathrm{O} 3^{\mathrm{a}}\right)=169^{\circ}($ symmetry $\operatorname{code} a=1 / 2+\mathrm{x}, 1 / 2-\mathrm{y}, \mathrm{z})$.

\section{Spectral Data}

\section{Diethyl 2-(3-oxo-1,3-di(thiophen-2-yl)propyl)malonate (3a)}

White solid; yield 82\%; mp 58-60 ${ }^{\circ} \mathrm{C} ;{ }^{1} \mathrm{H}-\mathrm{NMR}\left(400 \mathrm{MHz}, \mathrm{CDCl}_{3}\right) \delta 7.76(\mathrm{~d}, J=4.0$ $\mathrm{Hz}, 1 \mathrm{H}), 7.62(\mathrm{~d}, J=4.8 \mathrm{~Hz}, 1 \mathrm{H}), 7.13-7.10(\mathrm{~m}, 2 \mathrm{H}), 6.93(\mathrm{~d}, J=3.2 \mathrm{~Hz}, 1 \mathrm{H}), 6.86(\mathrm{dd}, J=$ $4.8,4.0 \mathrm{~Hz}, 1 \mathrm{H}), 4.51$ (q, $J=7.4 \mathrm{~Hz}, 1 \mathrm{H}), 4.26-4.16(\mathrm{~m}, 2 \mathrm{H}), 4.07$ (q, $J=7.0 \mathrm{~Hz}, 2 \mathrm{H}), 3.88$ $(\mathrm{d}, J=8.8 \mathrm{~Hz}, 1 \mathrm{H}), 3.49(\mathrm{~d}, J=6.8 \mathrm{~Hz}, 2 \mathrm{H}), 1.25(\mathrm{t}, J=7.0 \mathrm{~Hz}, 3 \mathrm{H}), 1.90$ (t, $J=7.2 \mathrm{~Hz}, 3 \mathrm{H})$; ${ }^{13} \mathrm{C}-\mathrm{NMR}\left(100 \mathrm{MHz}, \mathrm{CDCl}_{3}\right) \delta 190.0,167.9,167.5,144.0,143.2,133.8,132.1,128.1,126.5$, 125.9, 124.2, 61.7, 61.5, 57.7, 43.8, 36.2, 14.0, 13.8; IR (KBr, $\left.\mathrm{cm}^{-1}\right)$ 3112, 3073, 2991, 2938, 2904, 2871, 1747, 1725, 1660, 1517, 1419, 1290, 1245, 1213, 1166, 1051, 1027, 862, 723, 690, 538; Elemental analysis (\%), Calcd for $\mathrm{C}_{18} \mathrm{H}_{20} \mathrm{O}_{5} \mathrm{~S}_{2}$ : C, 56.82; H, 5.30; S, 16.86; Found: C, 56.79; H, 5.27; S, 16.81 .

\section{Diethyl 2-(3-oxo-3-(thiophen-2-yl)-1-p-tolylpropyl)malonate (3b)}

White solid; yield 84\%; mp 67-70 ${ }^{\circ} \mathrm{C} ;{ }^{1} \mathrm{H}-\mathrm{NMR}\left(400 \mathrm{MHz}, \mathrm{CDCl}_{3}\right) \delta 7.73(\mathrm{~d}, J=3.6$ $\mathrm{Hz}, 1 \mathrm{H}), 7.54$ (d, $J=4.8 \mathrm{~Hz}, 1 \mathrm{H}), 7.15$ (d, $J=8.0 \mathrm{~Hz}, 2 \mathrm{H}), 7.07-7.02$ (m, 3H), 4.22-4.10 (m, $3 \mathrm{H}), 3.95(\mathrm{q}, J=7.0 \mathrm{~Hz}, 2 \mathrm{H}), 3.82(\mathrm{~d}, J=10.0 \mathrm{~Hz}, 1 \mathrm{H}), 3.45(\mathrm{dd}, J=16.0,4.4 \mathrm{~Hz}, 1 \mathrm{H}), 3.35$ $(\mathrm{dd}, J=16.0,9.2 \mathrm{~Hz}, 1 \mathrm{H}), 2.23(\mathrm{~s}, 3 \mathrm{H}), 1.23(\mathrm{t}, J=7.2 \mathrm{~Hz}, 3 \mathrm{H}), 1.00(\mathrm{t}, J=7.2 \mathrm{~Hz}, 3 \mathrm{H}) ;{ }^{13} \mathrm{C}-$ NMR (100 MHz, $\left.\mathrm{CDCl}_{3}\right) \delta 190.5,168.3,167.7,144.1,137.0,136.6,133.7,132.1,129.0$ (2C), 128.1, 128.0 (2C), 61.6, 61.3, 57.5, 43.4, 40.8, 21.0, 14.0, 13.7; IR (KBr, cm $\left.{ }^{-1}\right) 3104,2981$, $2904,1725,1662,1517,1419,1259,1230,1162$, 1027, 858, 728, 547; Elemental analysis (\%), Calcd for $\mathrm{C}_{21} \mathrm{H}_{24} \mathrm{O}_{5} \mathrm{~S}: \mathrm{C}, 64.93 ; \mathrm{H}, 6.23$; S, 8.25; Found: C, 64.89; H, 6.20; S, 8.21. 


\section{Diethyl 2-(1-(4-methoxyphenyl)-3-oxo-3-(thiophen-2-yl)propyl)malonate (3c)}

White solid; yield 82\%; mp 79-82 ${ }^{\circ} \mathrm{C} ;{ }^{1} \mathrm{H}-\mathrm{NMR}\left(400 \mathrm{MHz}, \mathrm{CDCl}_{3}\right) \delta 7.74$ (d, $J=3.6$ $\mathrm{Hz}, 1 \mathrm{H}), 7.57$ (d, $J=4.8 \mathrm{~Hz}, 1 \mathrm{H}), 7.18$ (d, $J=8.4 \mathrm{~Hz}, 2 \mathrm{H}), 7.08$ (t, $J=4.4 \mathrm{~Hz}, 1 \mathrm{H}), 6.77$ (d, $J$ $=8.4 \mathrm{~Hz}, 2 \mathrm{H}), 4.23-4.16(\mathrm{~m}, 2 \mathrm{H}), 4.12(\mathrm{dt}, J=9.7,4.6 \mathrm{~Hz}, 1 \mathrm{H}), 3.96(\mathrm{q}, J=7.0 \mathrm{~Hz}, 2 \mathrm{H}), 3.79$ (d, $J=10.0 \mathrm{~Hz}, 1 \mathrm{H}), 3.72$ (s, 3H), 3.43 (dd, $J=16.0,4.4 \mathrm{~Hz}, 1 \mathrm{H}), 3.33$ (dd, $J=16.0,9.6 \mathrm{~Hz}$, $1 \mathrm{H}), 1.25(\mathrm{t}, J=7.2 \mathrm{~Hz}, 3 \mathrm{H}), 1.02(\mathrm{t}, J=7.2 \mathrm{~Hz}, 3 \mathrm{H}) ;{ }^{13} \mathrm{C}-\mathrm{NMR}\left(100 \mathrm{MHz}, \mathrm{CDCl}_{3}\right) \delta 190.5$, $168.3,167.7,158.5,144.1,133.7,132.1,132.0,129.2$ (2C), 128.1, 113.7 (2C), 61.6, 61.3, 57.6, 55.1, 43.5, 40.5, 14.0, 13.8; IR (KBr, cm $\left.{ }^{-1}\right)$ 3102, 2981, 2937, 2904, 2836, 1747, 1729, $1660,1612,1513,1415,1369,1249,1180,1153,1031,858,833,728,561$; Elemental analysis (\%), Calcd for $\mathrm{C}_{21} \mathrm{H}_{24} \mathrm{O}_{6} \mathrm{~S}$ : C, 62.36; H, 5.98; S, 7.93; Found: C, 62.32; H, 5.95; S, 7.90 .

\section{Diethyl 2-(3-(4-methoxyphenyl)-3-oxo-1-(thiophen-2-yl)propyl)malonate (3d)}

Viscous oil; yield 86\%; ${ }^{1} \mathrm{H}-\mathrm{NMR}\left(400 \mathrm{MHz}, \mathrm{CDCl}_{3}\right) \delta 7.94(\mathrm{~d}, J=8.4 \mathrm{~Hz}, 2 \mathrm{H}), 7.13$ (d, $J=4.8 \mathrm{~Hz}, 1 \mathrm{H}), 6.93(\mathrm{bd}, J=6.8 \mathrm{~Hz}, 3 \mathrm{H}), 6.87$ (d, $J=4.0 \mathrm{~Hz}, 1 \mathrm{H}), 4.54$ (dd, $J=14.4,7.0$ $\mathrm{Hz}, 1 \mathrm{H}), 4.24-4.17$ (m, 2H), 4.08 (q, $J=7.2 \mathrm{~Hz}, 2 \mathrm{H}), 3.89$ (bd, 1H), 3.87 (s, 3H), 3.52 (d, $J=$ $7.0 \mathrm{~Hz}, 2 \mathrm{H}), 1.26(\mathrm{t}, J=7.0 \mathrm{~Hz}, 3 \mathrm{H}), 1.13(\mathrm{t}, J=7.0 \mathrm{~Hz}, 3 \mathrm{H}) ;{ }^{13} \mathrm{C}-\mathrm{NMR}\left(100 \mathrm{MHz}, \mathrm{CDCl}_{3},\right) \delta$ 195.7, 168.1, 167.7, 163.5, 143.7, 130.4, 129.8, 126.5, 125.7, 124.1, 113.7, 61.6, 61.5, 57.9, 55.4, 42.8, 36.1, 14.0, 13.8; IR (KBr, cm $\left.{ }^{-1}\right)$ 3108, 2981, 2935, 2840, 1747, 1731, 1675, 1600, $1575,1509,1419,1369,1307,1259,1172,1029,835,703$; Elemental analysis (\%), Calcd for $\mathrm{C}_{21} \mathrm{H}_{24} \mathrm{O}_{6} \mathrm{~S}$ : C, 62.36; H, 5.98; S, 7.93; Found: C, 62.32; H, 5.97; S, 7.90.

\section{Diethyl 2-(3-(furan-2-yl)-3-oxo-1-(thiophen-2-yl)propyl)malonate (3e)}

White solid; yield 79\%; mp 76-79 ${ }^{\circ} \mathrm{C}$; ${ }^{1} \mathrm{H}-\mathrm{NMR}\left(400 \mathrm{MHz}, \mathrm{CDCl}_{3}\right) \delta 7.35(\mathrm{~m}, 1 \mathrm{H})$, $7.17(\mathrm{~d}, J=3.6 \mathrm{~Hz}, 1 \mathrm{H}), 7.09$ (d, $J=5.2 \mathrm{~Hz}, 1 \mathrm{H}), 6.90(\mathrm{~d}, J=3.2 \mathrm{~Hz}, 1 \mathrm{H}), 6.82$ (t, $J=4.2$ $\mathrm{Hz}, 1 \mathrm{H}), 6.48$ (t, $J=1.8 \mathrm{~Hz}, 1 \mathrm{H}), 4.47$ (dt, $J=8.8,4.8 \mathrm{~Hz}, 1 \mathrm{H}), 4.22-4.13$ (m, 2H), 4.03 (q, $J$ $=7.0 \mathrm{~Hz}, 2 \mathrm{H}), 3.84(\mathrm{~d}, J=8.8 \mathrm{~Hz}, 1 \mathrm{H}), 3.43(\mathrm{dd}, J=16.6,9.0 \mathrm{~Hz}, 1 \mathrm{H}), 3.34(\mathrm{dd}, J=16.6,5.0$

$\mathrm{Hz}, 1 \mathrm{H}), 1.22$ (t, $J=7.0 \mathrm{~Hz}, 3 \mathrm{H}), 1.09$ (t, $J=7.0 \mathrm{~Hz}, 3 \mathrm{H}) ;{ }^{13} \mathrm{C}-\mathrm{NMR}\left(100 \mathrm{MHz}, \mathrm{CDCl}_{3},\right) \delta$ $186.1,167.8,167.5,152.4,146.5,143.2,126.5,125.8,124.2,117.4,112.2,61.6,61.5,57.8$, 42.9, 35.7, 13.9, 13.8; IR (KBr, cm $\left.{ }^{-1}\right): 3126,3095,2994,2929,1747,1725,1670,1562,1471$, 1403, 1290, 1247, 1218, 1168, 1039, 1027, 914, 850, 771, 690, 595; Elemental analysis (\%), Calcd for $\mathrm{C}_{18} \mathrm{H}_{20} \mathrm{O}_{6} \mathrm{~S}$ : C, 59.33; H, 5.53; S, 8.80; Found: C, 59.30; H, 5.51; S, 8.78. 


\section{rel-(3R,4S)-Ethyl 2-oxo-4,6-di(thiophen-2-yl)-1,2,3,4-tetrahydropyridine-3-carboxylate}

(4a)

Colorless crystal; yield 85\%; mp 140-143 ${ }^{\circ} \mathrm{C} ;{ }^{1} \mathrm{H}-\mathrm{NMR}\left(400 \mathrm{MHz}, \mathrm{CDCl}_{3}\right) \delta 9.00(\mathrm{~s}$, $1 \mathrm{H}), 7.32(\mathrm{~d}, J=3.2 \mathrm{~Hz}, 1 \mathrm{H}), 7.27(\mathrm{~d}, J=5.2 \mathrm{~Hz}, 1 \mathrm{H}), 7.23(\mathrm{~d}, J=4.4 \mathrm{~Hz}, 1 \mathrm{H}), 7.04$ (t, $J=$ $4.2 \mathrm{~Hz}, 1 \mathrm{H}), 6.98(\mathrm{~d}, J=3.6 \mathrm{~Hz}, 1 \mathrm{H}), 6.96(\mathrm{~d}, J=3.6 \mathrm{~Hz}, 1 \mathrm{H}), 5.66(\mathrm{~d}, J=2.8 \mathrm{~Hz}, 1 \mathrm{H}), 4.67$ (dd, $J=9.6,4.0 \mathrm{~Hz}, 1 \mathrm{H}), 4.28-4.17(\mathrm{~m}, 2 \mathrm{H}), 3.76$ (d, $J=9.6 \mathrm{~Hz}, 1 \mathrm{H}), 1.24$ (t, $J=7.2 \mathrm{~Hz}, 3 \mathrm{H})$; ${ }^{13} \mathrm{C}-\mathrm{NMR}\left(100 \mathrm{MHz}, \mathrm{CDCl}_{3}\right) \delta 168.2,167.2,143.6,136.9,131.5,127.7,127.0,125.7,125.1$, 124.7, 124.2, 104.7, 61.7, 56.0, 37.2, 14.1; IR (KBr, cm $\left.{ }^{-1}\right)$ 3205, 3108, 3068, 2973, 2933, $1743,1679,1648,1477,1375,1249,1145,1031,842,759,703$, 692; Elemental analysis (\%), Calcd for $\mathrm{C}_{16} \mathrm{H}_{15} \mathrm{NO}_{3} \mathrm{~S}_{2}$ : C, 57.64; H, 4.53; N, 4.20; S, 19.23; Found: C, 57.59; H, 4.44; N, 4.10; S, 19.11; GC/MS (m/z)(\%) $261\left(\mathrm{M}^{+}, 100\right), 200$ (34.69), 110 (63.94), 45 (34.36).

rel-(3R,4S)-Ethyl 2-oxo-6-(thiophen-2-yl)-4-p-tolyl-1,2,3,4-tetrahydropyridine-3carboxylate (4b)

Colorless crystal; yield 88\%; mp 186-189 ${ }^{\circ} \mathrm{C} ;{ }^{1} \mathrm{H}-\mathrm{NMR}\left(400 \mathrm{MHz}, \mathrm{CDCl}_{3}\right) \delta 8.77(\mathrm{~s}$, 1H), 7.26 (bt, $J=5.6 \mathrm{~Hz}, 2 \mathrm{H}), 7.21$ (d, $J=8.4 \mathrm{~Hz}, 2 \mathrm{H}), 7.16$ (d, $J=8.4 \mathrm{~Hz}, 2 \mathrm{H}), 7.04$ (dd, $J=$ 5.0, 3.8 Hz, 1H), $5.57(\mathrm{~d}, J=2.8 \mathrm{~Hz}, 1 \mathrm{H}), 4.34(\mathrm{dd}, J=10.4,4.0 \mathrm{~Hz}, 1 \mathrm{H}), 4.23-4.12(\mathrm{~m}, 2 \mathrm{H})$, $3.71(\mathrm{~d}, J=10.8 \mathrm{~Hz}, 1 \mathrm{H}), 2.35(\mathrm{~s}, 3 \mathrm{H}), 1.19(\mathrm{t}, J=7.2 \mathrm{~Hz}, 3 \mathrm{H}) ;{ }^{13} \mathrm{C}-\mathrm{NMR}\left(100 \mathrm{MHz}, \mathrm{CDCl}_{3}\right)$ $\delta 168.6,167.6,137.5,137.2(2 \mathrm{C}), 131.2,129.6$ (2C), 127.7, 127.5 (2C), 125.4, 123.7, 105.8, 61.5, 55.5, 41.6, 21.0, 14.0; IR (KBr, cm $\left.{ }^{-1}\right)$ 3193, 3099, 2977, 2904, 2863, 1737, 1673, 1650, 1511, 1473, 1371, 1263, 1160, 1029, 811, 707; Elemental analysis (\%), Calcd for $\mathrm{C}_{19} \mathrm{H}_{19} \mathrm{NO}_{3} \mathrm{~S}$ : C, 66.84; H, 5.61; N, 4.10; S, 9.39; Found: C, 66.79; H, 5.57; N, 4.04; S, 9.34; GC/MS (m/z)(\%) $269\left(\mathrm{M}^{+}, 100\right), 164$ (64.55), 110 (66.90), 91 (38.81).

\section{rel-(3R,4S)-Ethyl}

4-(4-methoxyphenyl)-2-oxo-6-(thiophen-2-yl)-1,2,3,4tetrahydropyridine-3-carboxylate (4c)

Colorless crystal; yield 91\%; mp 194-197 ${ }^{\circ} \mathrm{C} ;{ }^{1} \mathrm{H}-\mathrm{NMR}\left(400 \mathrm{MHz}, \mathrm{CDCl}_{3}\right) \delta 8.56(\mathrm{~s}$, 1H), 7.29-7.22 (m, 4H), $7.03(\mathrm{dd}, J=4.8,4.0 \mathrm{~Hz}, 1 \mathrm{H}), 6.88$ (d, $J=8.8 \mathrm{~Hz}, 2 \mathrm{H}), 5.56$ (bd, $J=$ $2.4 \mathrm{~Hz}, 1 \mathrm{H}), 4.32$ (dd, $J=10.6,3.8 \mathrm{~Hz}, 1 \mathrm{H}), 4.23-4.12(\mathrm{~m}, 2 \mathrm{H}), 3.81$ (s, 3H), 3.68 (d, $J=10.8$ $\mathrm{Hz}, 1 \mathrm{H}), 1.19$ (t, $J=7.2 \mathrm{~Hz}, 3 \mathrm{H}) ;{ }^{13} \mathrm{C}-\mathrm{NMR}\left(100 \mathrm{MHz}, \mathrm{CDCl}_{3}\right) \delta 168.6,167.4,158.9,137.2$, 132.5, 131.2, 128.7 (2C), 127.7, 125.4, 123.7, 114.2 (2C), 105.9, 61.5, 55.7, 55.3, 41.3, 14.1; IR $\left(\mathrm{KBr}, \mathrm{cm}^{-1}\right)$ 3191, 3118, 3099, 2979, 2929, 2830, 1739, 1673, 1644, 1511, 1471, 1371, 1249, 1151, 1039, 827, 715, 549; Elemental analysis (\%), Calcd for $\mathrm{C}_{19} \mathrm{H}_{19} \mathrm{NO}_{4} \mathrm{~S}$ : C, 63.85; 
H, 5.36; N, 3.92; S, 8.97; Found: C, 63.78; H, 5.29; N, 3.87; S, 8.91; GC/MS (m/z)(\%) 285 $\left(\mathrm{M}^{+}, 100\right), 256$ (39.07), 121 (67.73), $110(58.49)$.

\section{rel-(3R,4S)-Ethyl}

6-(4-methoxyphenyl)-2-oxo-4-(thiophen-2-yl)-1,2,3,4-

tetrahydropyridine-3-carboxylate (4d)

Colorless crystal; yield 89\%; mp 135-138 ${ }^{\circ} \mathrm{C}$; ${ }^{1} \mathrm{H}-\mathrm{NMR}\left(400 \mathrm{MHz}, \mathrm{CDCl}_{3}\right) \delta 7.86$ (s, $1 \mathrm{H}), 7.42(\mathrm{~d}, J=8.4 \mathrm{~Hz}, 2 \mathrm{H}), 7.22(\mathrm{bd}, J=4.8 \mathrm{~Hz}, 1 \mathrm{H}), 6.98-6.92(\mathrm{~m}, 4 \mathrm{H}), 5.51$ (bd, $J=4.0$ $\mathrm{Hz}, 1 \mathrm{H}), 4.65(\mathrm{dd}, J=9.0,4.2 \mathrm{~Hz}, 1 \mathrm{H}), 4.28-4.15$ (m, 2H), 3.84 (s, 3H), 3.74 (d, $J=9.2 \mathrm{~Hz}$, $1 \mathrm{H}), 1.23(\mathrm{t}, J=7.0 \mathrm{~Hz}, 3 \mathrm{H}) ;{ }^{13} \mathrm{C}-\mathrm{NMR}\left(100 \mathrm{MHz}, \mathrm{CDCl}_{3}\right) \delta 168.4,166.8,160.4,144.1$, 136.6 (2C), 127.0, 126.6 (2C), 125.0, 124.6, 114.3 (2C), 104.0, 61.7, 56.1, 55.4, 37.3, 14.1; IR $\left(\mathrm{KBr}, \mathrm{cm}^{-1}\right)$ 3203, 3102, 2973, 2933, 2838, 1737, 1681, 1650, 1606, 1515, 1378, 1292, 1245, 1147, 1027, 823, 769, 701, 514; Elemental analysis (\%), Calcd for $\mathrm{C}_{19} \mathrm{H}_{19} \mathrm{NO}_{4} \mathrm{~S}: \mathrm{C}, 63.85 ; \mathrm{H}$, 5.36; N, 3.92; S, 8.97; Found: C, 63.80; H, 5.30; N, 3.85; S, 8.92; GC/MS (m/z)(\%) $285\left(\mathrm{M}^{+}\right.$, 100), 188 (56.47), 134 (61.77), 91 (27.81).

rel-(3R,4S)-Ethyl 6-(furan-2-yl)-2-oxo-4-(thiophen-2-yl)-1,2,3,4-tetrahydropyridine-3carboxylate (4e)

Viscous oil; yield 75\%; ${ }^{1} \mathrm{H}-\mathrm{NMR}\left(400 \mathrm{MHz}, \mathrm{CDCl}_{3}\right) \delta 8.57$ (s, $\left.1 \mathrm{H}\right), 7.44$ (d, $J=0.8$ $\mathrm{Hz}, 1 \mathrm{H}), 7.23$ (dd, $J=4.8,0.8 \mathrm{~Hz}, 1 \mathrm{H}), 6.98-6.94$ (m, 2H), 6.67 (d, $J=3.2 \mathrm{~Hz}, 1 \mathrm{H}), 6.46$ (dd, $J=3.2,2.0 \mathrm{~Hz}, 1 \mathrm{H}), 5.78(\mathrm{bd}, J=4.0 \mathrm{~Hz}, 1 \mathrm{H}), 4.69$ (dd, $J=9.4,4.2 \mathrm{~Hz}, 1 \mathrm{H}), 4.28-4.19$ (m, $2 \mathrm{H}), 3.75(\mathrm{~d}, J=9.6 \mathrm{~Hz}, 1 \mathrm{H}), 1.24(\mathrm{t}, J=7.2 \mathrm{~Hz}, 3 \mathrm{H}) ;{ }^{13} \mathrm{C}-\mathrm{NMR}\left(100 \mathrm{MHz}, \mathrm{CDCl}_{3}\right) \delta 168.2$, 166.6, 147.0, 143.7, 142.9, 128.1, 126.9, 125.0, 124.7, 111.6, 106.7, 102.9, 61.8, 56.1, 37.0, 14.0; IR (KBr, $\left.\mathrm{cm}^{-1}\right)$ 3226, 3116, 2979, 2935, 2871, 1735, 1683, 1496, 1373, 1263, 1157, 1024, 740, 703; Elemental analysis (\%), Calcd for $\mathrm{C}_{16} \mathrm{H}_{15} \mathrm{NO}_{4} \mathrm{~S}_{2}: \mathrm{C}, 60.55 ; \mathrm{H}, 4.76 ; \mathrm{N}, 4.41$; S, 10.10; Found: C, 60.47; H, 4.68; N, 4.35; S, 10.03; GC/MS (m/z)(\%) $245\left(\mathrm{M}^{+}, 100\right), 216$ (33.94), 148 (25.63), 94 (32.15).

\section{4,6-Di(thiophen-2-yl)-3,4-dihydropyridin-2(1H)-one (5a)}

Brown crystal; yield 58\%; mp 172-175 ${ }^{\circ} \mathrm{C} ;{ }^{1} \mathrm{H}-\mathrm{NMR}\left(400 \mathrm{MHz}, \mathrm{CDCl}_{3}\right) \delta 7.67$ (s, 1H), $7.30(\mathrm{~d}, J=5.2 \mathrm{~Hz}, 1 \mathrm{H}), 7.22(\mathrm{~d}, J=4.8 \mathrm{~Hz}, 1 \mathrm{H}), 7.20$ (d, $J=3.6 \mathrm{~Hz}, 1 \mathrm{H}), 7.07$ (dd, $J=$ 4.8, 4.0 Hz, 1H), 7.00-6.95 (m, 2H), 5.69 (bd, $J=4.4 \mathrm{~Hz}, 1 \mathrm{H}), 4.27-4.21$ (m, 1H), 2.98 (dd, $J$ $=16.0,6.8 \mathrm{~Hz}, 1 \mathrm{H}), 2.83(\mathrm{dd}, J=16.0,8.4 \mathrm{~Hz}, 1 \mathrm{H}) ;{ }^{13} \mathrm{C}-\mathrm{NMR}\left(100 \mathrm{MHz}, \mathrm{CDCl}_{3}\right) \delta 169.9$, 145.8, 137.6, 131.6, 127.7, 127.0, 125.6, 124.1, 123.9, 123.4, 105.6, 39.4, 33.7; IR (KBr, cm

$\left.{ }^{1}\right) 3210,3097,2958,2927,2854,1725,1679,1646,1463,1378,1297,973,786,721,698$; Elemental analysis (\%), Calcd for $\mathrm{C}_{13} \mathrm{H}_{11} \mathrm{NOS}_{2}$ : C, 59.74; H, 4.24; N, 5.36; S, 24.54; Found: 
C, 59.66; H, 4.09; N, 5.30; S, 24.45; GC/MS (m/z)(\%) $261\left(\mathrm{M}^{+}, 100\right), 200(34.20), 110$ (63.13), 45 (32.90).

\section{6-(Thiophen-2-yl)-4-p-tolyl-3,4-dihydropyridin-2(1H)-one (5b)}

Brown crystal; yield 65\%; mp 143-146 ${ }^{\circ} \mathrm{C} ;{ }^{1} \mathrm{H}-\mathrm{NMR}\left(400 \mathrm{MHz}, \mathrm{CDCl}_{3}\right) \delta 8.46(\mathrm{~s}$, $1 \mathrm{H}), 7.28(\mathrm{~d}, J=2.8 \mathrm{~Hz}, 1 \mathrm{H}), 7.26(\mathrm{~d}, J=4.8 \mathrm{~Hz}, 1 \mathrm{H}), 7.21$ (d, $J=8.2 \mathrm{~Hz}, 2 \mathrm{H}), 7.18$ (d, $J=$ $8.2 \mathrm{~Hz}, 2 \mathrm{H}), 7.06$ (dd, $J=5.0,3.8 \mathrm{~Hz}, 1 \mathrm{H}), 5.62$ (d, $J=3.6 \mathrm{~Hz}, 1 \mathrm{H}), 3.97-3.90$ (m, 1H), 2.88 $(\mathrm{dd}, J=16.3,7.2 \mathrm{~Hz}, 1 \mathrm{H}), 2.72(\mathrm{dd}, J=16.3,10.2 \mathrm{~Hz}, 1 \mathrm{H}), 2.37$ (s, 3H); ${ }^{13} \mathrm{C}-\mathrm{NMR}(100$ $\mathrm{MHz}, \mathrm{CDCl}_{3}$,) $\delta 171.1,139.7,137.9,136.7,131.6,129.6$ (2C), 127.7, 127.0 (2C), 125.1, 123.3, 106.4, 39.1, 38.2, 21.0; IR ( $\left.\mathrm{KBr}, \mathrm{cm}^{-1}\right)$ 3197, 3104, 2998, 2935, 2842, 1681, 1644, $1509,1473,1369,1249,935,815,715,505$; Elemental analysis (\%), Calcd for $\mathrm{C}_{16} \mathrm{H}_{15} \mathrm{NOS}$ : C, 71.34; H, 5.61; N, 5.20; S, 11.90; Found: C, 71.27; H, 5.55; N, 5.13; S, 11.79; GC/MS (m/z)(\%) $269\left(\mathrm{M}^{+}, 100\right), 164$ (65.58), 110 (65.20), 91 (37.42).

\section{4-(4-Methoxyphenyl)-6-(thiophen-2-yl)-3,4-dihydropyridin-2(1H)-one (5c)}

Brown crystal; yield 68\%; mp 123-126 ${ }^{\circ} \mathrm{C}$; ${ }^{1} \mathrm{H}-\mathrm{NMR}\left(400 \mathrm{MHz}, \mathrm{CDCl}_{3}\right) \delta 7.90$ (s, $1 \mathrm{H}), 7.27(\mathrm{~d}, J=4.8 \mathrm{~Hz}, 1 \mathrm{H}), 7.22(\mathrm{~d}, J=8.6 \mathrm{~Hz}, 2 \mathrm{H}), 7.21-7.20(\mathrm{~m}, 1 \mathrm{H}), 7.06(\mathrm{dd}, J=4.8$, $3.6 \mathrm{~Hz}, 1 \mathrm{H}), 6.90$ (d, $J=8.6 \mathrm{~Hz}, 2 \mathrm{H}), 5.61$ (d, $J=3.6 \mathrm{~Hz}, 1 \mathrm{H}), 3.95-3.89(\mathrm{~m}, 1 \mathrm{H}), 3.82$ (s, $3 \mathrm{H}), 2.87(\mathrm{dd}, J=16.1,7.0 \mathrm{~Hz}, 1 \mathrm{H}), 2.70(\mathrm{dd}, J=16.1,10.0 \mathrm{~Hz}, 1 \mathrm{H}) ;{ }^{13} \mathrm{C}-\mathrm{NMR}(100 \mathrm{MHz}$, $\left.\mathrm{CDCl}_{3}\right) \delta 170.6,158.6,137.9,134.7,131.4,128.1$ (2C), 127.7, 125.2, 123.1, 114.3 (2C), 106.6, 55.3, 39.2, 37.8; IR (KBr, $\left.\mathrm{cm}^{-1}\right)$ 3205, 3097, 2991, 2902, 2829, 1679, 1644, 1610, $1511,1461,1365,1247,1035,823$, 701; Elemental analysis (\%), Calcd for $\mathrm{C}_{16} \mathrm{H}_{15} \mathrm{NO}_{2} \mathrm{~S}$ : C, 67.34; H, 5.30; N, 4.91; S, 11.24; Found: C, 67.26; H, 5.23; N, 4.88; S, 11.16; GC/MS $(\mathrm{m} / \mathrm{z})(\%) 285\left(\mathrm{M}^{+}, 100\right), 256(39.48), 121$ (72.17), 110 (63.07).

\section{6-(4-Methoxyphenyl)-4-(thiophen-2-yl)-3,4-dihydropyridin-2(1H)-one (5d)}

Brown crystal; yield 62\%; mp 122-125 ${ }^{\circ} \mathrm{C} ;{ }^{1} \mathrm{H}-\mathrm{NMR}\left(400 \mathrm{MHz}, \mathrm{CDCl}_{3}\right) \delta 7.41$ (bd, $J$ $=8.8 \mathrm{~Hz}, 3 \mathrm{H}), 7.21(\mathrm{~d}, J=4.0 \mathrm{~Hz}, 1 \mathrm{H}), 7.00-6.92(\mathrm{~m}, 4 \mathrm{H}), 5.53(\mathrm{~d}, J=3.6 \mathrm{~Hz}, 1 \mathrm{H}), 4.23(\mathrm{dd}$, $J=11.6,8.0 \mathrm{~Hz}, 1 \mathrm{H}), 3.85(\mathrm{~s}, 3 \mathrm{H}), 2.96(\mathrm{dd}, J=16.0,6.8 \mathrm{~Hz}, 1 \mathrm{H}), 2.81(\mathrm{dd}, J=16.0,8.4 \mathrm{~Hz}$, $1 \mathrm{H}) ;{ }^{13} \mathrm{C}-\mathrm{NMR}\left(100 \mathrm{MHz}, \mathrm{CDCl}_{3}\right) \delta 170.3,160.3,146.5,136.8,127.2,126.9,126.5(2 \mathrm{C})$, 123.9, 123.7, 114.3 (2C), 104.7, 55.3, 39.4, 33.7; IR (KBr, $\left.\mathrm{cm}^{-1}\right)$ 3193, 3097, 3014, 2956, 2931, 2832, 1673, 1644, 1610, 1513, 1371, 1286, 1240, 1029, 829, 767, 721; Elemental analysis (\%), Calcd for $\mathrm{C}_{16} \mathrm{H}_{15} \mathrm{NO}_{2} \mathrm{~S}: \mathrm{C}, 67.34 ; \mathrm{H}, 5.30 ; \mathrm{N}, 4.91 ; \mathrm{S}, 11.24$; Found: $\mathrm{C}, 67.22$; H, 5.24; N, 4.84; S, 11.18; GC/MS (m/z)(\%) $285\left(\mathrm{M}^{+}, 100\right), 188$ (55.29), 134 (59.10), 91 (26.23). 